\title{
Capturing plasticity effects in overdriven shocks on the finite scale
}

\author{
Scott R. Runnels \\ Los Alamos National Laboraotory, P.O. Box 1663, Los Alamos, NM 87545 \\ SRunnels@LANL.gov \\ 505-500-2842
}

\begin{abstract}
An ordinary differential equation (ODE) form of the radial return algorithm is combined with a strain-rate hardening model to produce an ODE that describes deviatoric stress through a prescribed density rise. An analytical solution is found to the resulting ODE for a specific choice of one of the hardening model's parameters. That solution is used to prove that if the prescribed density rise is allowed to be infinitely thin, i.e., like a shock in the mathematical sense, the resulting deviatoric stress is still bounded. In other words, the singularity is integrable; integration of the radial return ODE regularizes the infinite strain rate and resulting yield stress in the presence of an ideal shock singularity. The analytical tools developed for this line of thinking are applied to study the variation of deviatoric stress through a nearly shock-like density rise using different density rise profiles, revealing the impact of the shape choice. The tools are also used to compute what rise times are needed to converge upon the correct value of deviatoric stress through a shock; the results indicate that most contemporary hydrocodes cannot be expected to achieve those rise times. A demonstration of connecting the analytical tools to a hydrocode, using surrogate numerical shock shapes, is provided thereby opening the door for using such surrogates to perform sub-grid computations of converged shock behavior for strain-rate hardening materials.
\end{abstract}

Keywords: shocks, plasticity, hardening, hydrodynamics, radial return

\section{Introduction}

This article is concerned with the structure of over-driven plastic shocks in metals, how that structure is related to deviatoric stress through the shocks, and 
algorithms used for continuum scale computations, i.e., "hydrocodes". Shock structure is a topic that has received significant attention from different vantage points during the past sixty years. One vantage point of particular importance is that of experimentation. As discussed in the review article [3], various highresolution shock wave measurement methods have been used over several decades to study geomaterials and metals. Strength anomalies, phase transformation, thermoplastic deformation, fracture and other plasticity effects can be at least partially discerned in measured shock profiles, and that understanding can be augmented with modeling.

From a different vantage point, shock structure has been studied using continuum and sub-continuum mechanics models, augmented with phenomenological material mdoels. One excellent example is [8], which proposes an analytical solution for the shape of plastic shocks in the non-overdriven case where an elastic precursor precedes the plastic wave. The authors use a power-law yield stress model for the material with physical laws on the continuum scale and a viscoplastic flow rule that introduces a relaxation time scale, thereby leading to a shock structure. Key to their model is the introduction of a physical time scale. In the situation of overdriven shocks considered here where the rate-independent radial return method is used with a fully saturated yield stress, no such time scales enter directly. Yet a finite rise time and physics of rapid compression are considered when developing the yield stress model that will be used here, in particular the thinking in [20] and [21], which contributes to [13].

From another vantage point, the study of shock structure focuses on results produced by discretized continuum-scale computations in hydrocodes. While there are examples of using continuum approaches to resolve the shock structure [16], typically spatial discretization is too coarse to resolve that structure in application. Therefore, in the hydrocode context, the shock is typically viewed as a mathematical singularity where, by definition, the state variables change instantaneously. The very important Rankine-Hugoniot equations are emblematic of that thinking [14] [12], and that same line of reasoning even forms the theoretical basis of many hydro schemes, i.e., Godunov-like methods, which are based on solving the Riemann problem at cell boundaries. Eulerian and cell-centered Lagrangian hydrocode methods have been based on that idea; see for example [17], [5] and [1]. On the other hand, staggered grid Lagrangian hydro methods do not incorporate the notion of the shock singularity directly in their algorithms, so instead they must use artificial viscosity, an idea that was perhaps first introduced from a more intuitive standpoint [19], but has since been recognized as necessary for satisfying thermodynamic conditions across the shock [9]. 
In the hydrocode context, the mathematics associated with spatial and temporal discretization play an important role in the structure of the computed shock. It is perhaps the unwanted influence of mathematical discretization errors that amplifies the notion of the singular shock as an ideal that stands in contrast to the more dissipative shocks that hydrocodes typically deliver. The pursuit of the idealized shock, which is in a way a shock without structure, is served in large part by the hydrocode community's historical connections to verification problems that do not involve material strength. In those verification problems, e.g., [11] [15], the shock is a mathematical singularity in a gas and it is often the goal of hydrocode methods to converge toward that singularity. As such, there is an intense focus on dissipation mechanisms, both numerical and physical, wanted as well as unwanted. The proper use of Godunov methods and artificial viscosity, e.g., limiters, has received a great deal of attention, again, often with the notion of convergence to the shock as a mathematical singularity. Even when considering hydrocode performance on plastic shocks, the notion of the idealized singularity carries over. An example is in [18], in which the underdriven shock is idealized. Although it stands in contrast to [8] and [16], the approach in [18] leads to a convenient analytical solution, complete with an ideal elastic precursor and an ideal plastic wave following, that is helpful in the verification of elastic perfectly plastic hydrocode simulations.

To some extent, the different viewpoints come full circle wherein hydrocodes are used to study the effects of shock structure in experiments, for example [23]. In fact, numerous examples of modeling used to design and interpret experiments exists. However, as can be discerned from the above discussion, the elements from the different viewpoints on shocks together form a conceptual mismatch between how shocks are viewed. In particular, the perfectly sharp, idealized shocks sought by hydro algorithm developers, the nearly sharp shocks assumed by those who develop strength models, and the reality of relatively gentle shocks actually produced by modern hydrocodes provide a mixture of ideas that are not always aligned. In particular, this three-way mismatch undermines the idea of material strength model validation in the presence of different shock hydro treatments, including mesh size choices and artificial viscosity values, and is therefore a mismatch worth addressing.

In this article, an attempt is made at bridging some of the conceptual gaps regarding overdriven plastic shock structure by combining two essential ideas and analyzing them together. The first idea is the radial return algorithm, which is what many hydrocodes use to handle plasticity. In a very rough sense, the radial return algorithm operates in a projection and then correction mode. It is easily 
described by assuming that the stress state is already on the yield surface and that deformation is continuing to increase. In that situation, the radial return method makes a projection, using a simple elastic model, as to what the next time step's stress state will be, given the current strain rate. Then, acknowledging that stress states outside the yield surface cannot exist, it scales back the stress tensor so that it lies on the yield surface. It is in the projection step that the first conceptual mismatch enters. Specifically, if a hydrocode were to achieve an ideal shock, the strain rate at that point would be infinite.

The second essential idea is the model for the yield stress, which is an input into the radial return method. In regimes of high deformation rate, it is likely that the yield stress is strain-rate dependent, and that is where the conceptual mismatch is further highlighted. If one were to achieve a perfectly sharp shock, an infinite strain rate would lead to an infinite projected trial value and also, in principle, an infinite value for the yield stress as well. Of course, that infinite yield stress would only exist for an infinitessimal time for a particular piece of material. So the question is whether or not that singularity is integrable in the context of radial return and, if so, what relationship does the regularized, finite value have with reality.

As this study shows analytically and numerically, it appears that that the singularity is, in fact, integrable and regularized by the radial return algorithm. In other words, it appears based on the analysis herein, that if one could achieve a perfectly sharp shock, then even with a strain-rate based hardening model, a finite stress state would result. What this study also shows, however, is that the rise time needed to converge on the correct value of deviatoric stress is far smaller than what any contemporary hydrocode can be expected to provide and is smaller than the physical mechanisms considered in developing the models used. By combining the analysis provided in this article with a hydrocode, it may be possible to bridge that gap and provide converged solutions for the deviatoric stress on meshes that would otherwise be too coarse to capture that converged value. The primary aim of this article is to demonstrate the need for such an approach and also open the door to that way of thinking.

\section{ODE form of radial return}

The first part of this analysis relies upon the work of Margolin and Flower who provided an ordinary differential equation (ODE) representation of the radial return method [6]. A derivation of that ODE is summarized here, using a slightly different yield criteria than in [6] to be consistent with the criteria often used in 
staggered-grid radial return algorithms. The elastic projection of the deviatoric stress $s_{i j}$ over time increment $\delta t$ is the first step of the radial return method,

$$
s_{j k}^{*}=s_{j k}^{n}+2 G \dot{e}_{j k} \delta t
$$

where the $n$ superscript indicates evaluation at time step $t_{n}, G$ is the elastic shear modulus, $\dot{e}_{j k}$ is the time rate of change of the deviatoric strain, and $s_{j k}^{*}$ is the intermediate, projected trial value of the deviatoric stress. That trial stress is scaled to produce a final stress state that lies on the yield surface. The stress invariant

$$
\begin{aligned}
J_{2} & \equiv \frac{1}{6}\left[\left(s_{11}-s_{22}\right)^{2}+\left(s_{22}-s_{33}\right)^{2}+\left(s_{33}-s_{11}\right)^{2}\right] \\
& +s_{12}^{2}+s_{23}^{2}+s_{31}^{2} \\
& =\frac{s_{i j} s_{j i}}{2}
\end{aligned}
$$

with the yield stress $Y$ in the yield condition

$$
\frac{\sqrt{3 J_{2}}}{Y}=1
$$

defines the yield surface. The above condition is used to compute a scaling factor for reducing the trial value so that the resulting deviatoric stress lies on the yield surface, i.e.,

$$
s_{j k}^{n+1}=s_{j k}^{*} \sqrt{\frac{(2 / 3) Y^{2}\left(t^{n}+\delta t\right)}{s_{j k}^{*} s_{j k}^{*}}}
$$

Adding Equations 1 and 4, rearranging, and dividing by the time increment $\delta t$ produces

$$
\frac{s_{j k}^{n+1}-s_{j k}^{n}}{\delta t}=\frac{s_{j k}^{*}}{\delta t}\left[\sqrt{\frac{(2 / 3) Y^{2}\left(t^{n}+\delta t\right)}{s_{j k}^{*} s_{j k}^{*}}}-1\right]+2 G \dot{e}_{j k}
$$

The ODE version of the algorithm is derived by seeking the limit as $\delta t \rightarrow 0$. Application of L'Hopital's rule and use of a Taylor series expansion for $\dot{Y}$ produces the ODE

$$
\frac{d s_{j k}}{d t}=2 G \dot{e}_{j k}+\frac{s_{j k}^{n}}{Y} \dot{Y}-s_{j k}^{n} \frac{3 G s_{m n} \dot{e}_{n m}}{Y^{2}}
$$




\section{1-D case with strain-rate hardening}

This investigation focuses on one-dimensional (1-D) planar shocks where all components of the strain tensor $\varepsilon_{i j}$ are zero except for $\varepsilon_{11}$. The off-diagonal components of the deviatoric strain tensor $e_{i j}$ are also zero while the diagonal entries are $e_{11}=(2 / 3) \varepsilon_{11}$ and $e_{22}=e_{33}=-(1 / 3) \varepsilon_{11}$. The product in the left-most term of Equation 6 is greatly simplified by observing that $s_{22}=s_{33}=-s_{11} / 2$, the latter part of which can be proven by following the radial return method through the projection and scaling steps described above. Thus, the 1-D planar shock version of the radial return method is

$$
\dot{s}=\frac{4}{3} G \dot{\varepsilon}+s \frac{\dot{Y}}{Y}-s^{2} 3 \dot{\varepsilon} \frac{G}{Y^{2}}
$$

where the " 11 " subscripts on $s$ and $\varepsilon$ have been dropped for convenience.

A prescribed density profile $\rho=\rho(t)$ will be used here as a means for studying plasticity and strain-rate based hardening through a density rise; this rise will be manipulated and eventually thought of as a shock in multiple senses of the word. The initial value for density is $\rho(0)=\rho_{0}$ and in this 1 -D case,

$$
\begin{gathered}
\varepsilon=1-\frac{\rho_{0}}{\rho} \\
\dot{\varepsilon}=\rho_{0} \frac{\dot{\rho}}{\rho^{2}} \\
\ddot{\varepsilon}=\frac{\rho_{0}}{\rho^{2}}\left[\ddot{\rho}-2 \frac{\dot{\rho}^{2}}{\rho}\right]
\end{gathered}
$$

Overdriven shocks in metals are added to this analysis next. The density rise time for such materials is likely in the picosecond to nanosecond time frame. As stated in [13], the yield stress is saturated in that case and may be expressed as

$$
Y=\frac{s_{o}}{\gamma^{\beta}} G\left(\frac{\dot{e_{p}}}{\dot{\xi}}\right)^{\beta}
$$

Here $e_{p}$ is the equivalent plastic strain, $\beta$ is a material constant that is approximately $0.25, s_{O}$ and $\gamma$ are also material constants, and $\xi$ is

$$
\xi=\frac{1}{2}\left(\frac{4 \pi \rho}{3 M}\right)^{1 / 3}\left(\frac{G}{\rho}\right)^{1 / 2}
$$


where $M$ is the molecular weight. In [13], the yield stress is predicted by taking the minimum of predictions provided by two separate rules; this is done to address the transition between thermally-driven shocks and strongly over-driven shocks. In this analysis, since some simplification is needed to pursue an analytical solution, the hardening rule above is used alone. For simplicity, the above hardening rule will be referred to here as the "PTW model", in reference to its authors" last names, again recognizing that it is only part of their model, somewhat simplified here to enable analytical results. Also, total volumetric strain will be used as an approximation for the plastic strain, i.e., $e_{p} \approx \frac{1}{3} \varepsilon$. Making that substitution into the expression for $\xi$, produces

$$
Y=K \rho^{\beta / 6} \dot{\varepsilon}^{\beta}
$$

where the constants have been coalesced into $K$, which is defined as

$$
K \equiv\left[\frac{s_{o}}{\gamma^{\beta}}\left(G^{1-\beta / 2}\right)\left(\frac{2}{3}\right)^{\beta}\left(\frac{4 \pi}{3 M}\right)^{-\beta / 3}\right]
$$

\section{Analytical solution}

In the case of a density rise that is linear in time, an analytical solution for the deviatoric stress through time can be obtained. For this 1-D case, a linearly varying density profile may be represented as $\rho(t)=\rho_{0}+r t$, where $r$ is the rate at which density rises over time; one may think of it, for example as $r=\left(\rho_{1}-\rho_{0}\right) D / w$ where $w$ is the width of the density rise, $D$ is the speed with which the disturbance transverses the material, and $\rho_{1}$ is the final density. It is a straightforward exercise to write down the time derivatives of this linear density rise and use them to form expressions for $\varepsilon, \dot{\varepsilon}, Y$, and $\dot{Y}$ with the equations given above. Inserting them into Equation 7, the radial return ODE for deviatoric stress becomes

$$
\dot{s}=\frac{4}{3} G \frac{\rho_{0} \dot{\rho}}{\rho^{2}}-s\left(\frac{11}{6}\right) \beta \frac{\dot{\rho}}{\rho}-s^{2} 3 \frac{G}{K^{2}}\left(\rho_{0} \dot{\rho}\right)^{1-2 \beta} \rho^{11 \beta / 3-2}
$$

It is possible to obtain an analytical solution to the above ODE for a special choice of $\beta=6 / 11$, a value that is admittedly high but, as subsequent results show, results in stress transients that are similar in nature to those produced by lower values, i.e., they are qualitatively the same. Making that choice for $\beta$ reduces the ODE 
to a general Ricatti equation that can be converted to a second-order ODE by two changes of variables, $v \equiv s C$ where

$$
C \equiv-\frac{3 G}{K^{2}}\left(\rho_{0} r\right)^{-1 / 11}
$$

and then $v \equiv-\dot{u} / u$. The solution of the resulting second-order ODE for $u$ is simply

$$
u(t)=C_{1}\left(\rho_{0}+r t\right)^{\alpha}+C_{2}\left(\rho_{0}+r t\right)^{-\alpha}
$$

where $C_{1}$ and $C_{2}$ are two constants of integration and

$$
\alpha \equiv\left(2 \frac{G}{K} \rho_{0}^{5 / 11}\right) r^{-6 / 11}
$$

Then, the solution for $s(t)$ is obtained by substitution of the change of variables,

$$
s(t)=-\frac{1}{C}(\alpha q) \frac{C_{1}\left(\rho_{0}+r t\right)^{\alpha-1}-C_{2}\left(\rho_{0}+r t\right)^{-\alpha-1}}{C_{1}\left(\rho_{0}+r t\right)^{\alpha}+C_{2}\left(\rho_{0}+r t\right)^{-\alpha}}
$$

The constants of integration are evaluated using initial conditions, which for this case are assumed to be $s(0)=0$ so that $v(0)=s(0) C=0$. The second change of variables, evaluated at $t=0$, means that $\dot{u}(0)=0$ so that

$$
C_{1} \rho_{0}^{\alpha-1}-C_{2} \rho_{0}^{-\alpha-1}=0
$$

from which the ratio of the two integration constants can be determined. Note that only the ratio is needed. Substitution into Equation 19 then produces

$$
s(t)=-\frac{1}{C}(\alpha r) \frac{\left(\rho_{0}+r t\right)^{\alpha-1}-\rho_{0}^{2 \alpha}\left(\rho_{0}+r t\right)^{-\alpha-1}}{\left(\rho_{0}+r t\right)^{\alpha}+\rho_{0}^{2 \alpha}\left(\rho_{0}+r t\right)^{-\alpha}}
$$

This analytical result may be used as a test case for the numerical integration of the radial return ODE along with the strain-rate hardening model. Verification of both the analytical solution and a numerical solution that integrates Equation 7 with auxiliary Equations 8-11 is shown in Figure 1 for multiple values of $K$, with $\beta=6 / 11$.

The next part of this analysis seeks to use the above analytical result to explore the case of an ideal, perfectly sharp shock as a limiting case of the linear density rise solution. To do so, it is convenient to express the density rate of change 


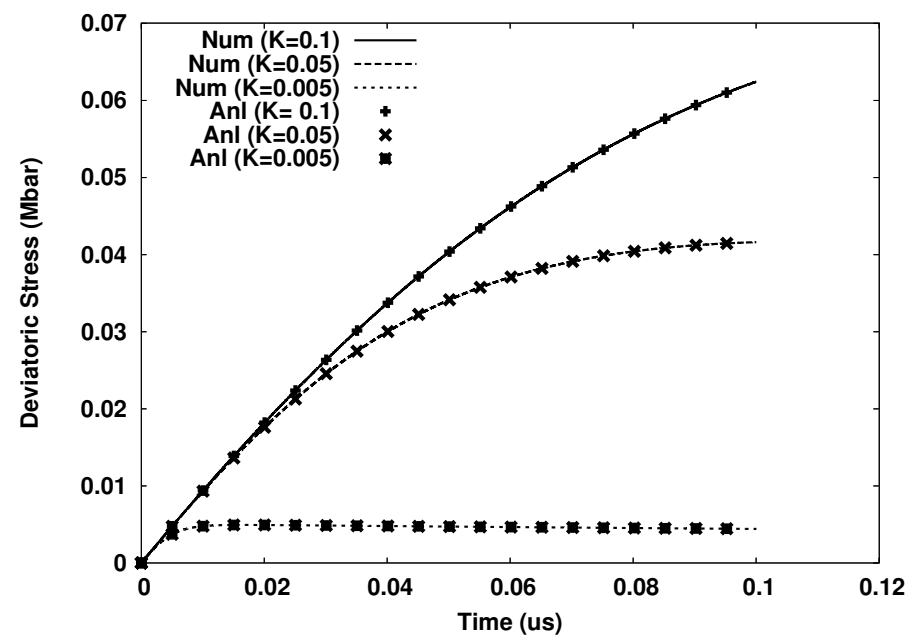

Figure 1: Verification of the numerical and analytical solutions to the radial return ODE with strain-rate hardening for various values of $K$; "Num" refers to the numerical integration of the equations while "Anl" refers to the analytical solution.

using a shock speed and width, i.e., $r=D \rho_{s} / w$, where $\rho_{s}=\rho_{1}-\rho_{0}$ is the density change. It is also convenient to express $\alpha$ in those same terms, i.e.,

$$
\alpha=\left[2 \frac{G}{K} \rho_{0}^{5 / 11} \rho_{s}^{-6 / 11}\right](w / D)^{6 / 11}
$$

where the term in the square brackets will henceforth be referred to as $\hat{d}$. The leading coefficient of the analytical solution evaluates to be

$$
-\frac{1}{C}(\alpha r)=\left[K \frac{2}{3}\left(\rho_{0} \rho_{s}\right)^{6 / 11}\right](w / D)^{-6 / 11}
$$

where the term in the square brackets will henceforth be referred to as $\hat{g}$. Making those substitutions while defining $s_{p}$ as the deviatoric stress at the end of the linear density rise, produces

$$
\begin{aligned}
& s_{p}=\hat{g}(w / D)^{-6 / 11} \times \\
& \frac{\rho_{1}^{\hat{d}(w / D)^{6 / 11}-1}-\rho_{0}^{2 \hat{d}(w / D)^{6 / 11}} \rho_{1}^{-\hat{d}(w / D)^{6 / 11}-1}}{\rho_{1}^{\hat{d}(w / D)^{6 / 11}}+\rho_{0}^{2 \hat{d}(w / D)^{6 / 11}} \rho_{1}^{-\hat{d}(w / D)^{6 / 11}}}
\end{aligned}
$$




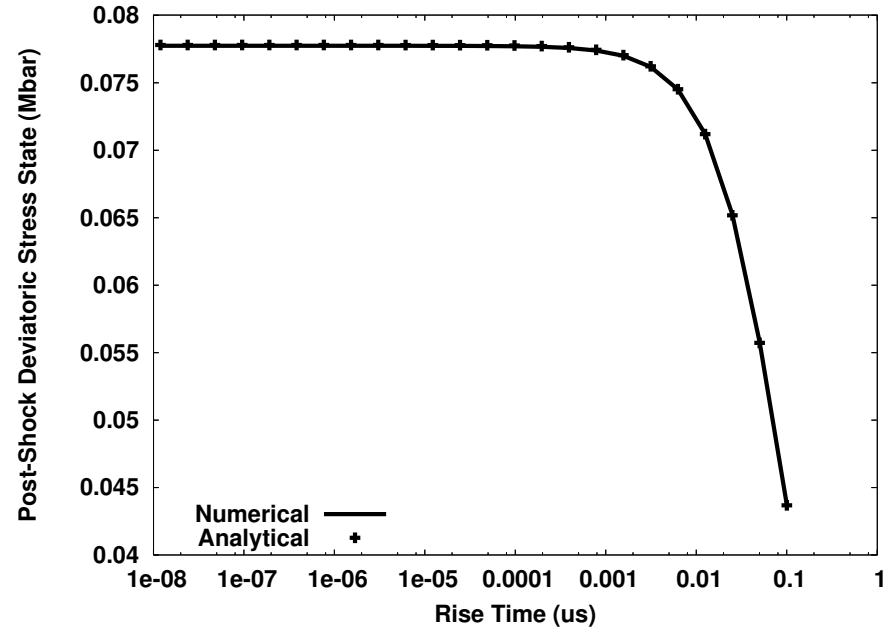

Figure 2: Numerical verification of the limiting process where the rise time $w / D$ is decreased exponentially, toward the limit $w / D \rightarrow 0$.

It is a straightforward matter to take the limit as $w / D \rightarrow 0$ of the above expression. Doing so produces,

$$
\hat{s}_{p} \equiv \lim _{w / D \rightarrow 0} s_{p}=\frac{4}{3} G \frac{\ln \left(\rho_{1} / \rho_{0}\right)}{\rho_{1} / \rho_{0}}
$$

This result is important because it demonstrates that a perfectly sharp, mathematically ideal shock, which produces an infinite strain rate for an infinitessimal time period, is regularized by the radial return method when the PTW strain-rate hardening model is used. The analytical result is verified numerically by evaluating the analytical solution for decreasing shock widths, as shown in Figure 2. This result also points to the importance of the density ratio, henceforth defined as $\rho_{r} \equiv \rho_{1} / \rho_{0}$.

\subsection{Exploring the linear shock}

The analytical solution derived above provides proof that, at least for $\beta=$ $6 / 11$, the deviatoric stress is convergent to a finite value in the presence of a mathematically ideal shock and a strain-rate hardening model as provided in PTW. Furthermore, the analytical solution provides a valuable verification tool for a numerical solution of the radial return ODE with the overdriven hardening model. This verification is critical because for cases where $w / D \rightarrow 0$, multiple singularities are at play, which can be seen by inspection of Equations $7-11$ when an infinite strain 
rate occurs. With the hope that the deviatoric stress will also be finite in a shock for other $\beta$ values and with the verification of the numerical integration in hand, excursions of $\beta \neq 6 / 11$ are studied here, numerically. In particular, $\beta=0.25$ is of interest, since that is the value for several real materials.

Referring back to the discussion in the introduction, it is worthwhile to specify terminology of shocks more exactly for this context. Here, the term "shock width" will be used, but in this case it will be used in a way that bridges the gap between the material modeling notion of a finite shock width and the numerical shock width of hydrocodes, which is orders of magnitude larger. Proceeding in this manner, the value of $w$, above, will be thought of as the "shock width." In some of the studies that follow, it will be varied from a fairly large value, which is representative of a typical hydrocode result, down to a very small value that approaches the limit $w \rightarrow 0$. Since it is actually the ratio of $w / D$ that matters, ultimately it is that ratio, "rise time", that will be used in much of the analysis that follows. Likewise, the term "post-shock value" refers to the value just as the density rise is completing. Lastly, because the density rises linearly with time, the term "linear shock" is used here in discussing the associated results, in contrast to the sinusoidally shaped shock considered later.

Computations are carried out for copper, nominally, with $\rho_{0}=8.93 \mathrm{~g} / \mathrm{cm}^{3}$, $G=0.48 \mathrm{Mbar}, M=1.055 \times 10^{-22}$ amu, $s_{0}=0.0092$, and $\gamma=8 \times 10^{-6}$. Figure 3 shows the deviatoric stress during the linear shock for different values of $\beta$ while Figure 4 shows how the post-shock deviatoric stress varies as the limit $w / D \rightarrow 0$ is approached numerically, for various values of $\beta$. The maximum deviatoric stress values during the linear shock are explored in more detail for a fixed $w / D$ value of $0.01 \mu \mathrm{s}$ in Figure 5, for different values of $\beta$ and $\rho_{r}$. Although not shown, a very similar trend is exhibited for the post-shock deviatoric stress. Figure 6 shows what values of $w / D$ are required to achieve approximate convergence to the hypothesized limiting value as $w / D \rightarrow 0$. As an example, in Figure 4 , the postlinear shock deviatoric stress state converges at a rise time of about $1 \times 10^{-6} \mu \mathrm{s}$ for $\beta=6 / 11$. That value as well as the other values plotted in Figure 6 are obtained by setting a convergence criteria on the change in deviatoric stress as $w / D$ is decreased. This result is of particular importance for multiple reasons. As can be seen from the plot, the $\beta$ parameter has a profound effect on the convergence of both the post-shock and maximum deviatoric stresses. For larger values of $\beta$, the post-shock and maximum deviatoric stress converges for rise times that are arguably well outside the reach of standard hydrocodes. But as $\beta$ is decreased, the situation worsens. The figure shows that extremely short rise times are required to resolve the maximum deviatoric stress state. It is very likely that any hydrocode 


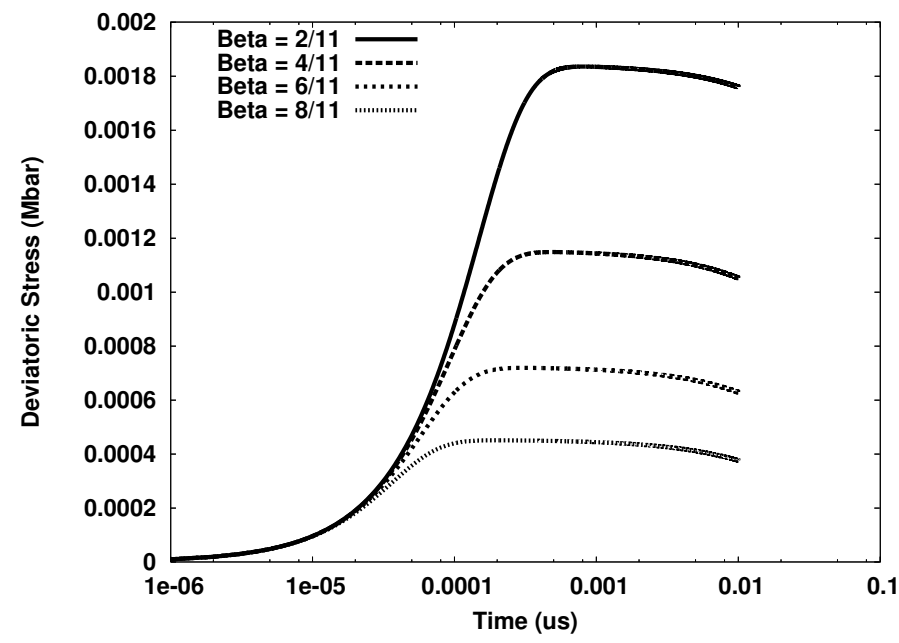

Figure 3: The transient deviatoric stress during a linear shock for various values of $\beta$.

simulation using gridding for practical applications will not be able to provide the necessary resolution to capture the deviatoric stress correctly.

\subsection{Sinusoidal shock profile}

The linear shock studied in the previous section is valuable because it is tied closely to the analytical solution, which also provides an analytical expression for the limiting case of $w / D \rightarrow 0$ when $\beta=6 / 11$. However, in contemplating how to apply this analysis to hydrocode simulations, it is worthwhile to consider other shapes that resemble hydrocode shocks more closely. Specifically, as discussed in [7], shocks on the finite scale are sinusoidal. While, for verification purposes, a sinusoidal density rise is not tied as closely to the analytical solution, the change in software required to implement a such a shape is trivial, those changes simply being that $\rho(t)=\rho_{0}+\rho_{s}(1-\cos \pi t D / w)$ instead of being linear, and that $\dot{\rho}$ and $\ddot{\rho}$ are provided accordingly.

The results for the sinusoidally varying shock are shown in in Figures 7 - 10 and correspond to the plots for the linear shock in Figures $3-6$. One aspect of the results stands out immediately, which is that the shape of the deviatoric transient during the sinusoidal shock is markedly different than that for the linear shock. That stated, the sinusoidally shaped shock produces very nearly the same predictions regarding rise time needed to capture the convergent deviatoric stress value. In particular, it is likely that contemporary hydrocodes in real applications would never obtain the converged value of the deviatoric stress in the shock. 


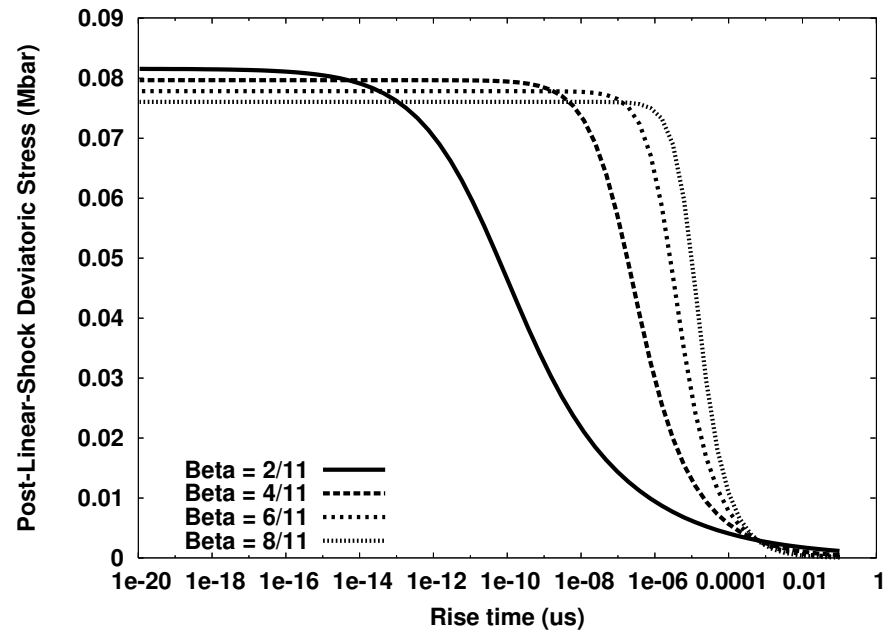

Figure 4: This result shows how the post-shock deviatoric stress varies as the limit $w / D \rightarrow 0$ is approached, for various values of $\beta$.

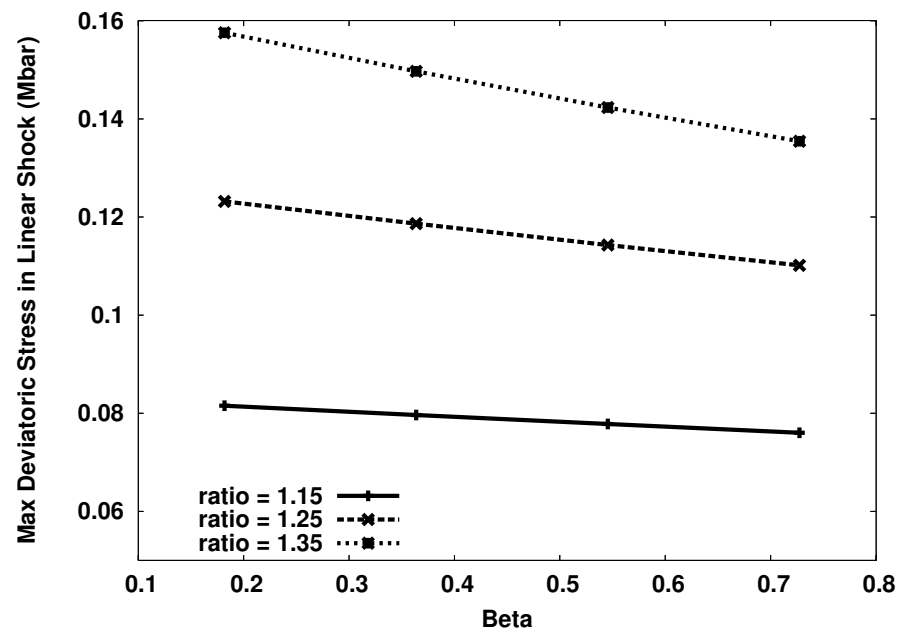

Figure 5: This figure shows the impact of $\beta$ and the post- to pre-shock density ratio on the maximum deviatoric stress experienced during the linear shock. 


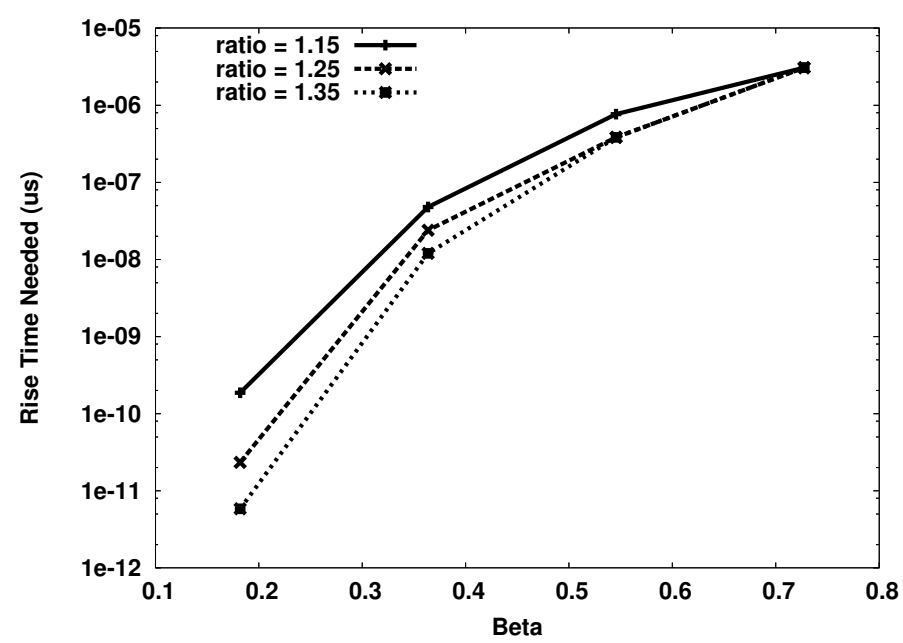

Figure 6: This figure shows what rise time is needed to converge upon the maximum deviatoric stress during the linear shock, i.e., what value of $w / D$ is needed such that smaller values produce essentially the same maximum result.

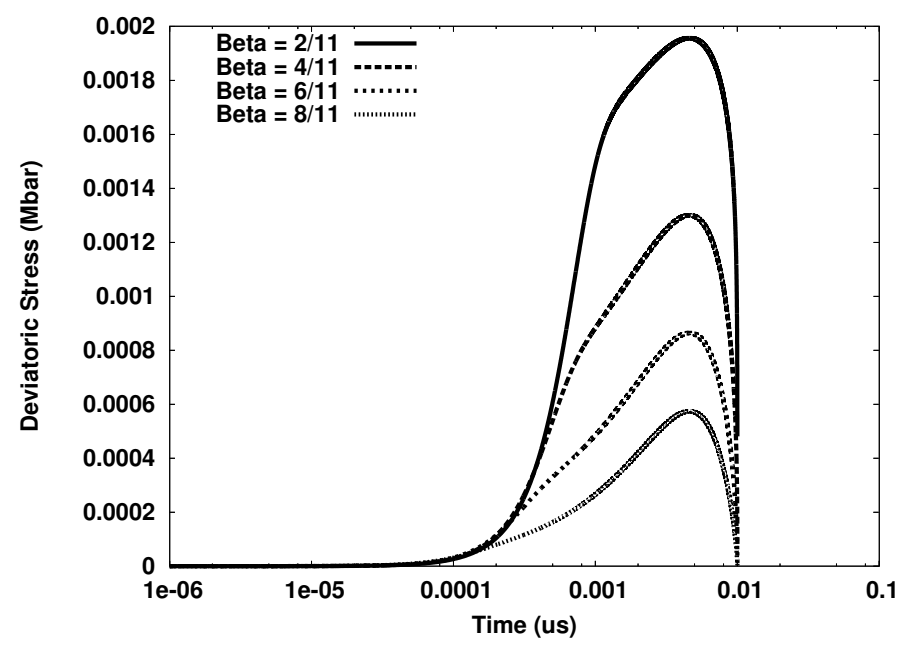

Figure 7: This figure shows the transient deviatoric stress during a sinusoidal shock for various values of $\beta$. 


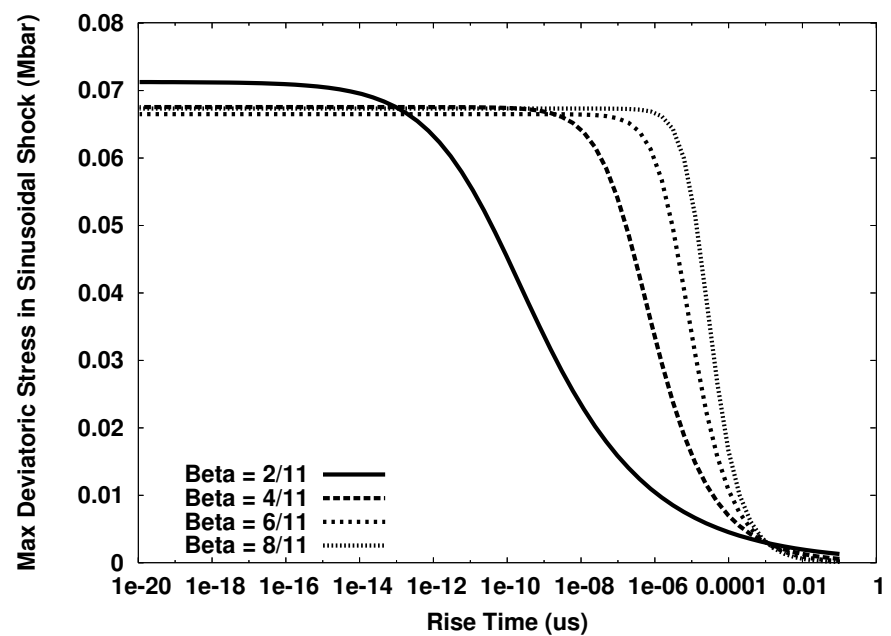

Figure 8: This result, which is for the sinusoidally shaped shock, shows how the post-shock deviatoric stress varies as the limit $w / D \rightarrow 0$ is approached, for various values of $\beta$.

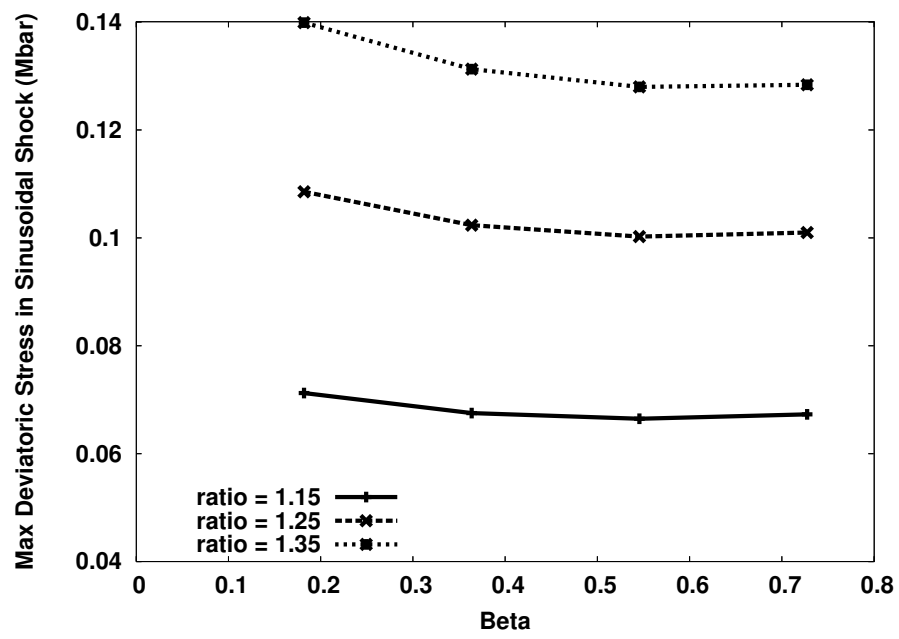

Figure 9: This figure shows the impact of $\beta$ and the post- to pre-shock density ratio on the maximum deviatoric stress experienced during the sinusoidal shock. 


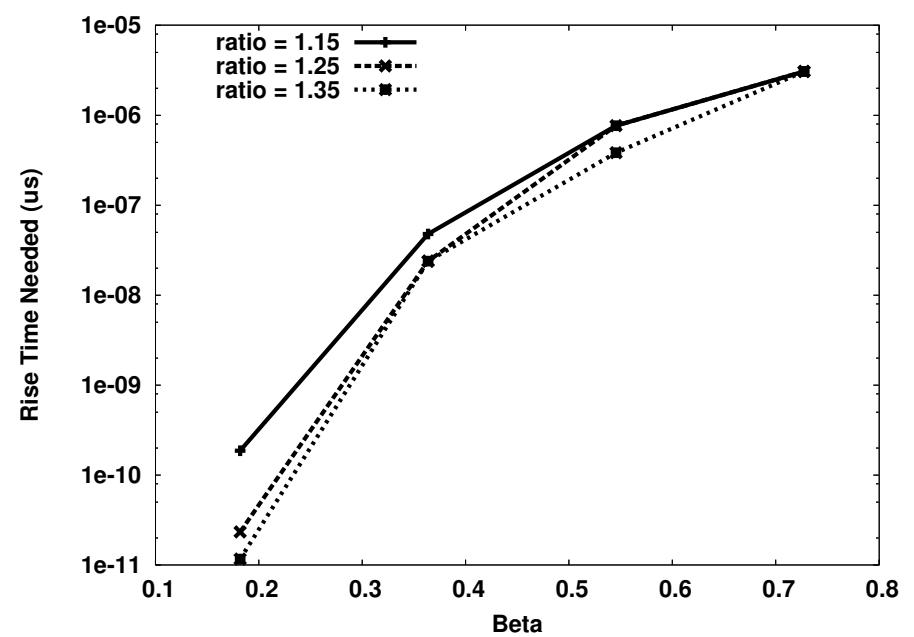

Figure 10: This figure shows what rise time is needed to converge upon the maximum deviatoric stress during the sinusoidal shock, i.e., what value of $w / D$ is needed such that smaller values produce essentially the same maximum result.

\section{Exploring traditional strain-rate hardening in a staggered grid hydro con- text}

Thus far, it has been proven that the radial return algorithm with the PTW hardening rule converges to a finite value in the presence of an ideal shock for $\beta=6 / 11$. The analytical solution for the radial return method with that hardening rule was used to verify numerical calculations. Then, numerical calculations were carried out for other values of $\beta, \rho_{r}$, and for two shock shapes (linear and sinusoidal). Results of the numerical integration indicate that excursions from $\beta=6 / 11$ to lower values reveals no new features when other parameters are held constant.

In this next section, the goal is to use the analytical and numerical methods promoted in the first part of this article as a lens through which to view what occurs during a standard staggered-grid hydro ("SGH") calculation of a plane wave, as in a piston problem. A mildly overdriven shock in copper is considered. The stress tensor in the material is given by $\sigma_{i j}=-p \delta_{i j}+2 G e_{i j}$. The bulk pressure $p$ is given by a Gruneisen equation of state [22], which also relies on the specific internal energy.

As before, this 1-D simulation will assume $G$ is constant and also the same relationships between total strain and deviatoric strain will hold, e.g., $e_{11}=\varepsilon_{11} 2 / 3$. 
For the plasticity calculations, the same invariant, yield, and scaling conditions of Equations 2, 3, and 4 will be used. For this simple case, $J_{2}$ reduces to $J_{2}=\frac{4}{3} G^{2} \varepsilon_{11}^{2}$. The piston problem initial conditions are an initial reference internal energy in the copper of 0 , an initial density of $8.93 \mathrm{~g} / \mathrm{cm}^{3}$, and an initial velocity of zero everywhere. The piston is represented by a velocity boundary condition in the left-most vertex moving at a velocity of $U$, which will be specified with the results.

The computations for an SGH simulation of the piston problem are performed using the "compatible" SGH algorithm. The theory is fully described in [2]; here it is briefly reviewed from an algorithmic standpoint for convenience. The 1-D simulation domain is divided into contiguous pieces called cells separated by vertices. These cells represent control volumes on which internal energy is evolved over time. An additional set of control volumes are centered around each vertex. Specifically, the control volume for a vertex extends between the center of mass of each adjacent cell. The algorithm advances the velocity of a vertex by summing the forces generated by the two cells touching the vertex's control volume and dividing by the vertex's control volume mass. The vertex's position is then advanced by integrating velocity. At the same time, the work required to accelerate the two vertices touching a cell is subtracted from that cell's internal energy. The Gruneisen equation of state provides the pressure and the radial return algorithm with PTW as described herein provides the deviatoric stresses. To them are added quadratic and linear artificial viscosity [4], computed from the velocity field.

The algorithm as described above would be first order in time. In fact a higher order, "leapfrog", temporal integration is used here in which the vertices and cells are advanced a half time-step, the resulting increase in energy, density, strain, and strain rate at that half time step is used to evaluate pressure and the rest of the stress tensor, and then that value for stress at the mid-time step is used to project the conditions at the beginning of the time step forward a full time step. The software used for computations was verified using the gas piston problem with a gamma-law gas (gamma $=5 / 3$ ) and the analytical solution to the elastic-perfectly plastic piston problem in [18].

\subsection{Radial return $O D E$ and $S G H$ performance}

In this section, numerical results are presented that point to the value of considering the type of analysis described in this article. Very highly refined SGH calculations are carried out for a piston problem with piston velocity $0.025 \mathrm{~cm} / \mathrm{us}$. Results are shown in Figures 11-14. Figure 11 demonstrates convergence with mesh refinement while Figure 12 demonstrates the difference in the shock shape with and without plasticity for two specific mesh refinements. From Figure 12 it 


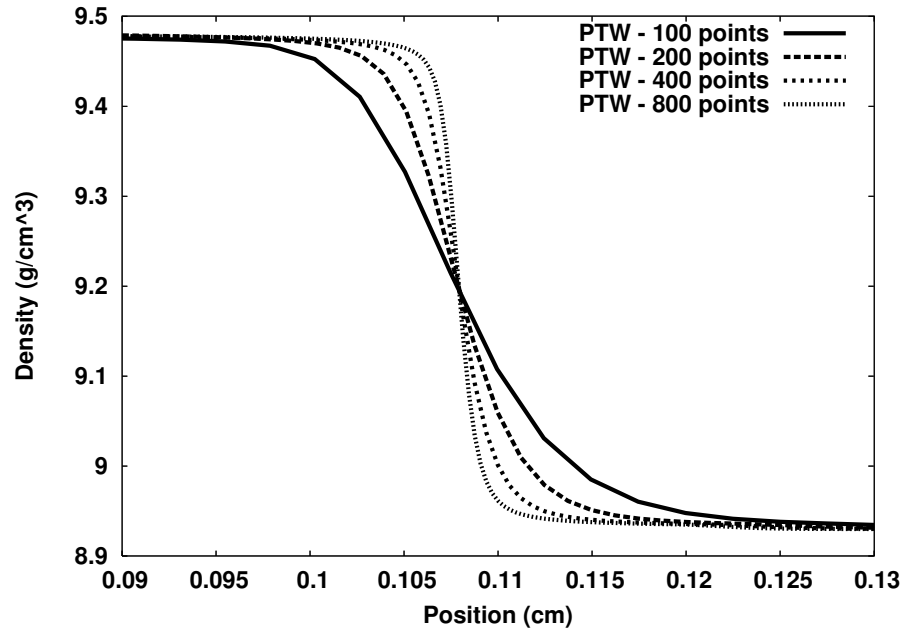

Figure 11: Spatial convergence of SGH with plasticity.

can be seen that the addition of plasticity to the otherwise pure gas piston problem does not significantly alter the position of the shock, if measured from the medium density. However, it does alter the position if measured from the shock completion or onset, which is perhaps a more reliable indicator [10]. Also, Figure 12 demonstrates that a significantly more diffused shock exists in the plastic calculation for the same refinement level. Therefore, while the inclusion of plasticity does not have a significant impact on the post-shock stress condition, which is dominated by the bulk pressure for this plane wave, its presence is clearly seen in the shape.

A more revealing aspect of the underlying numerics can be seen in Figures 13 and 14. As expected, the strain rate predicted numerically increases as the calculation is refined, as shown in Figure 13. Moreover, the strain rate is divergent as the grid is refined. It is worth noting that the integral above each of the curves shown in that figure are roughly equal, as shown in Figure 14. This is not unexpected, however; it points to the conclusion that the strain rate is approaching a Dirac delta, wherein its integral is constant. That fact plus the radial return's diffusive ability demonstrated in Figure 12 indicates that the radial return method regularizes the singularity in the SGH context. The fact that the radial return algorithm provides a regularizing effect in the overall numerical system can be exploited to bring closure to the various singularities associated with an ideal shock.

Next, the ODE version of the radial return algorithm is brought directly into the SGH context in two senses. First, SGH calculations are used to extract density 


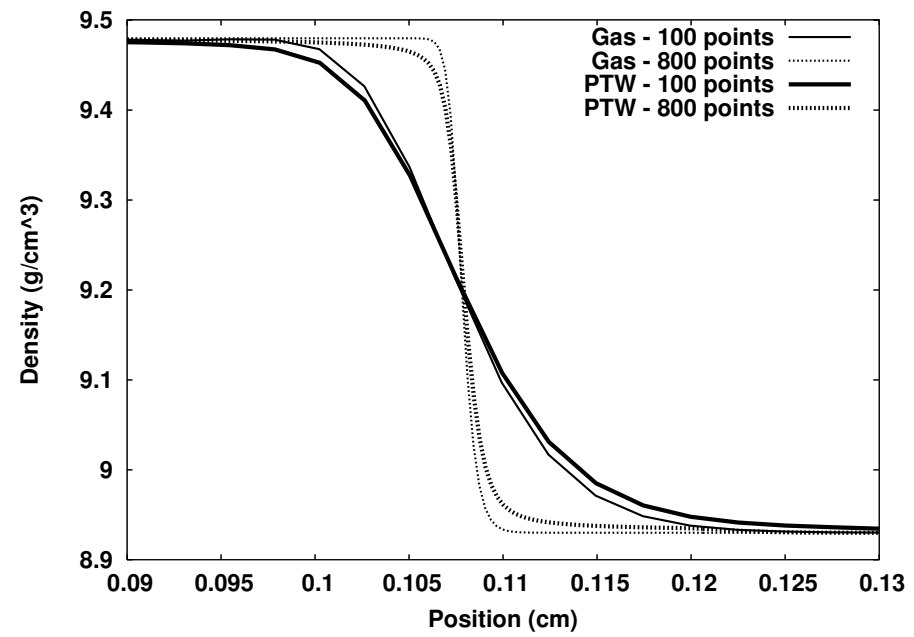

Figure 12: Comparison of SGH with and without plasticity; "Gas" means only the Gruneisen equation of state was used and "PTW" means that radial return with the PTW model for $Y$ was used.

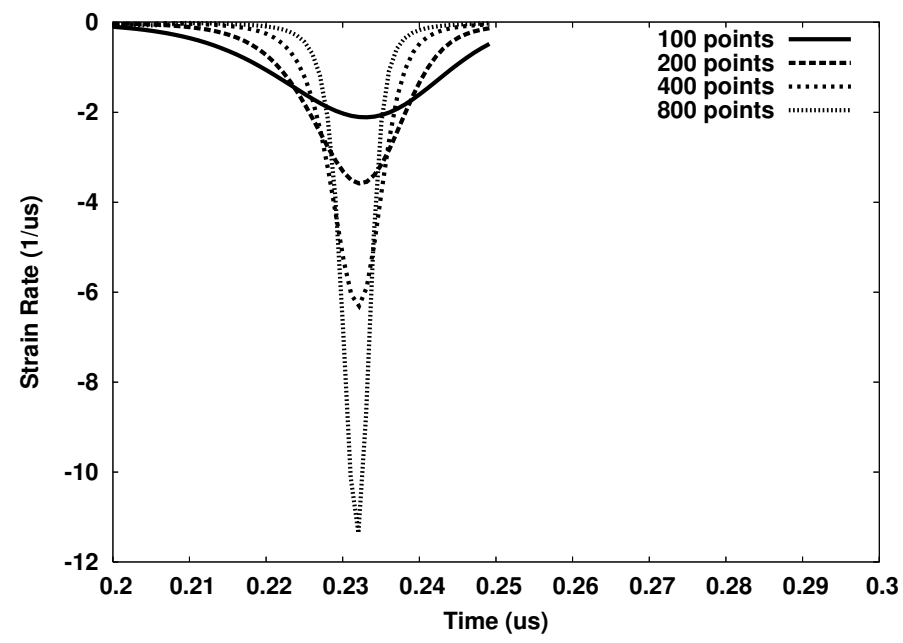

Figure 13: Strain rate during the spatial convergence study, exhibiting a Dirac delta behavior. 


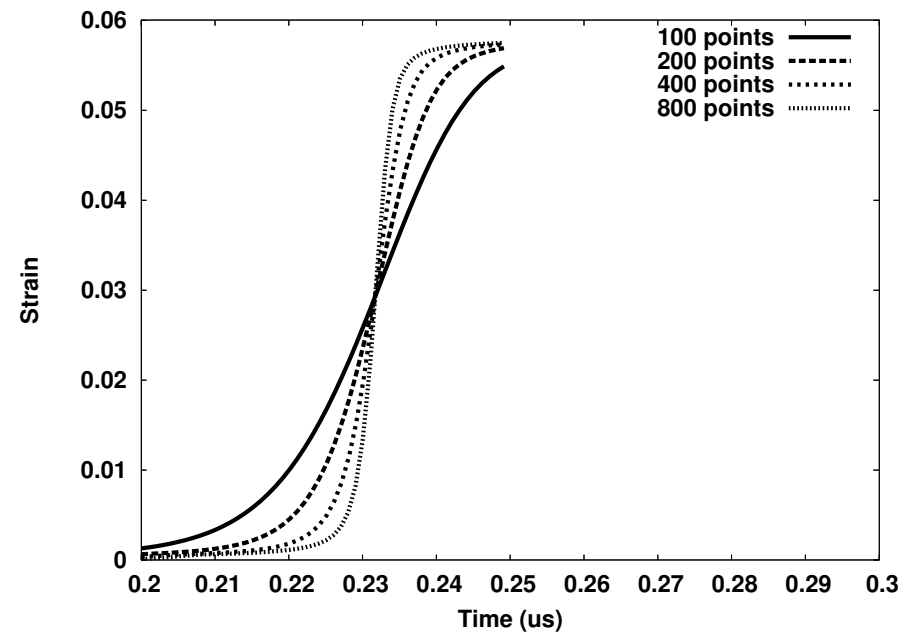

Figure 14: Strain through the SGH shock, which is constant for various refinements, pointing to the conclusion that the strain rate is behaving like a Dirac delta in the limit.

profiles that are fed into the analysis methods described earlier, and the results are overlaid on the SGH results. Figures 15 and 16 show those density profiles as linear and sinusoidal fits, respectively, each at different mesh resolutions. Henceforth, those fits will be referred to as "surrogate density profiles" and are used to represent the SGH density profiles as input to the radial return ODE. It is observed in Figures 17-20 that the numerical integration of the ODE using the surrogates can provide a reasonable approximation to the SGH radial return implementation, but only if the surrogates' shapes resemble the SGH density profile closely enough. For example, as shown in Figures 17 and 18 the linear surrogate clearly produces a deviatoric stress result that differs fairly significantly from the SGH result even under refinement. But at the same time, Figures 19 and 20 show that the sinusoidal surrogate provides a deviatoric stress transient that is more pleasing to the eye. These results are important because if an appropriate surrogate could be found, then a version of that surrogate representing a very small shock width, one small enough to converge on the deviatoric stress value, could be used for computing converged deviatoric stresses inside a mesh cell that contains the shock even though the mesh is too coarse to capture those converged values. In that vein of reasoning, it is worthwhile to note that although the sinusoidal surrogate provides more pleasing results than does the linear surrogate, it may not actually be the better choice, i.e., if the linear fit is more physically correct, it should perhaps be used instead. That stated, it is also worth noting that although the peaks produced 


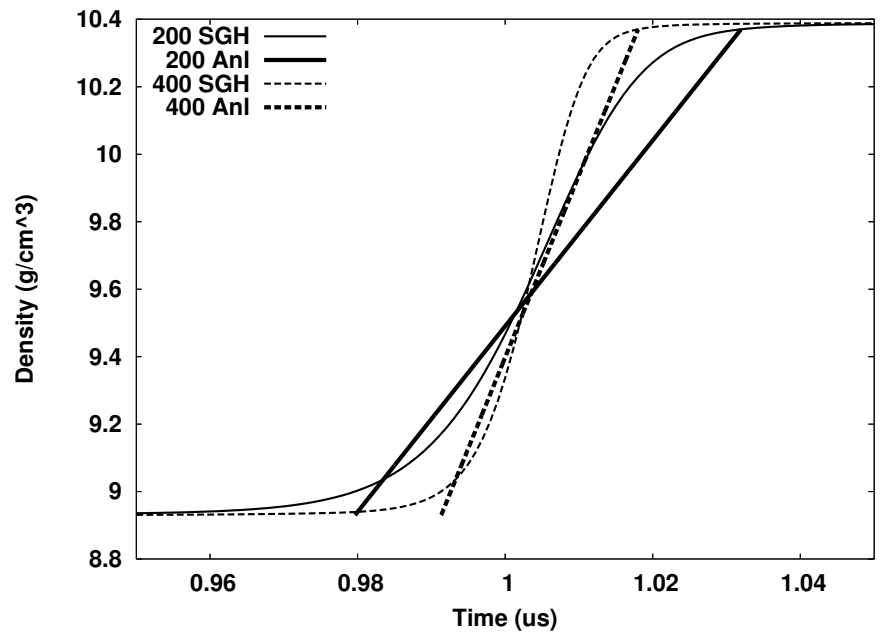

Figure 15: SGH density profiles with linear fits, or "surrogates", overlaid. "SGH" is the SGH density profile, "Anl" is the surrogate.

by the SGH and surrogates in Figures 19 and 20 have a very similar shape, the deviatoric stress produced by the SGH has a much more extended right foot, which is due to the numerical dissipation associated with the SGH method.

Also in this section, results are shown for when the radial return ODE is included directly in the SGH calculations, post-processing the values of strain, strain-rate, etc., to compute a deviatoric stress by numerical integration of the ODE. Figure 21 shows that the results of those two calculations are very close. However, it should be noted that in producing the numerically integrated ODE results, it was necessary to compute $\dot{Y}$ using a temporal finite difference of $Y$ as opposed to using the time derivatives of density as shown in Equations 8-10. If those functions based on density are used, very small fluctuations in densities cause large changes in its derivatives that pollute $Y$ and the deviatoric stress.

\section{Conclusions}

In the particular class of problems considered here, where the shock is overdriven and a rate independent radial return algorithm is used with a fully saturated yield stress model, a mismatch of concepts emerges around the notion of the shock. This mismatch occurs because there are no physical time scales directly associated with the shock, thereby causing the SGH calculations to tend toward a mathematically singular shock. Yet, in that same context, the yield stress 


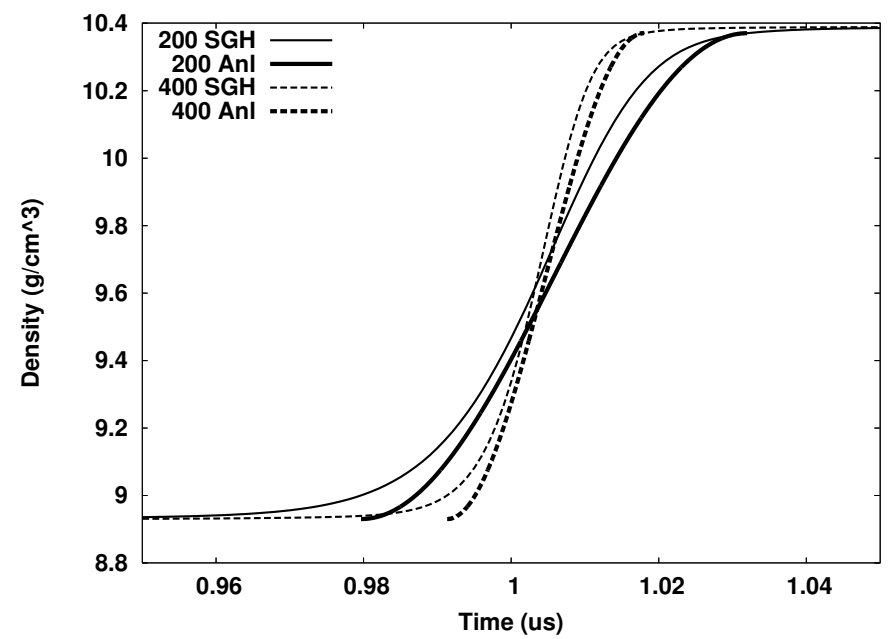

Figure 16: SGH density profiles with sinusoidal fits, or "surrogates", overlaid. "SGH" is the SGH density profile, "Anl" is the surrogate.

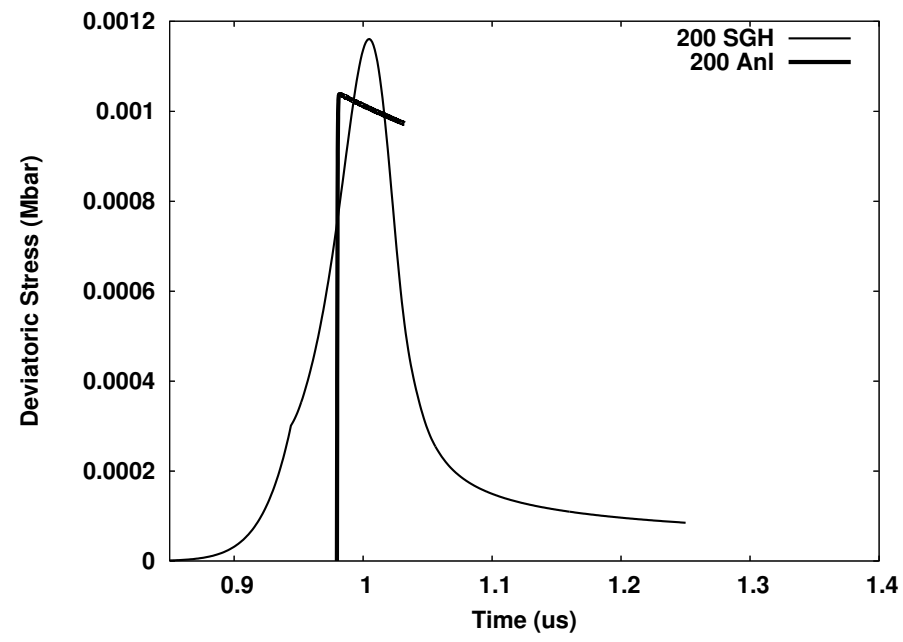

Figure 17: SGH deviatoric stress with corresponding integrated radial return values obtained using the linear surrogate, overlaid. "SGH" is the value produced by the SGH algorithm while "Anl" is the result produced by the surrogate with the radial return ODE. 


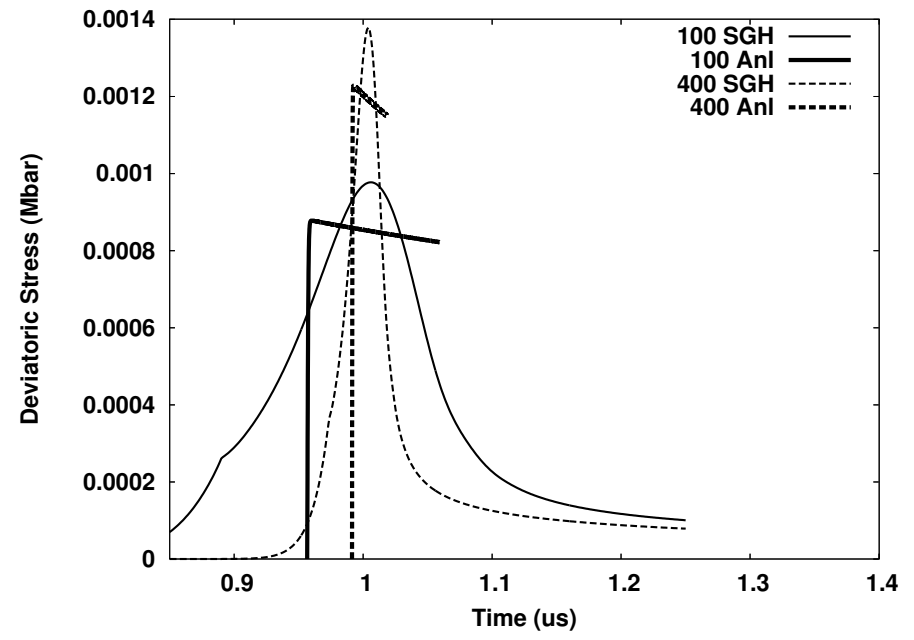

Figure 18: This figure shows results like that in Figure 17 but for other levels of grid refinement.

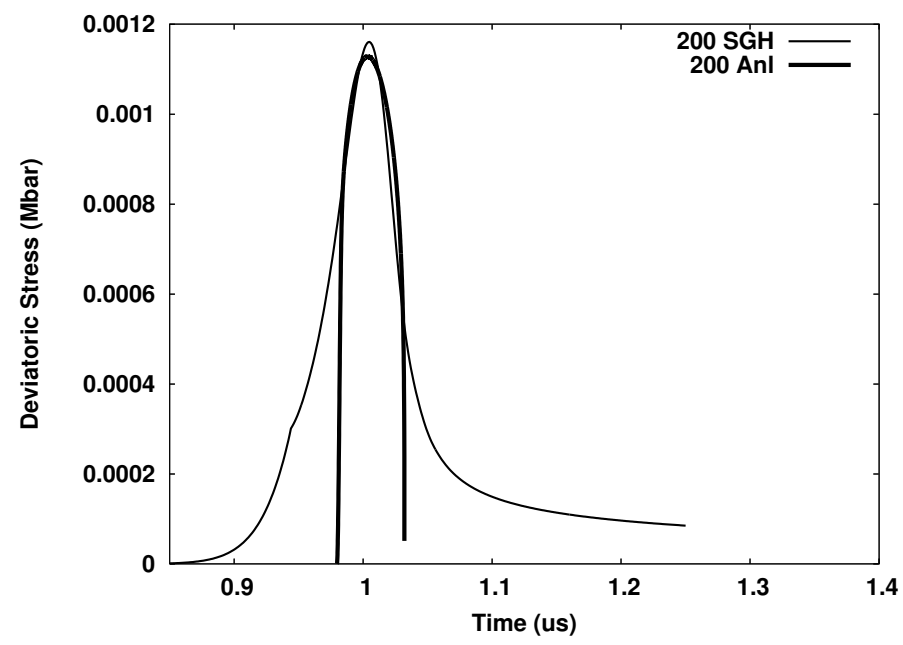

Figure 19: SGH deviatoric stress with corresponding integrated radial return values obtained using the sinusoidal surrogate, overlaid. "SGH" is the value produced by the SGH algorithm while "Anl" is the result produced by the surrogate with the radial return ODE. 


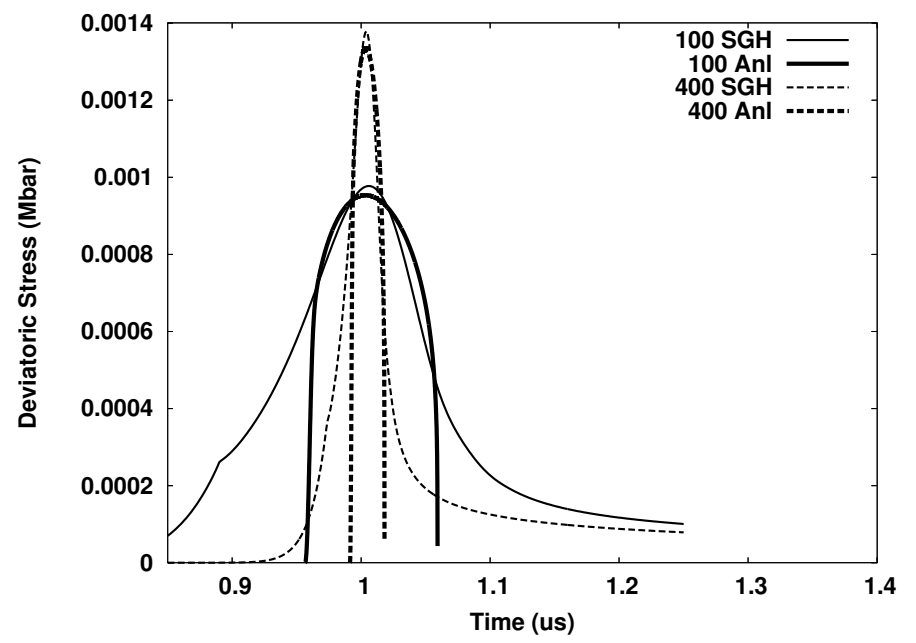

Figure 20: This figure shows results like that in Figure 19 but for other levels of grid refinement.

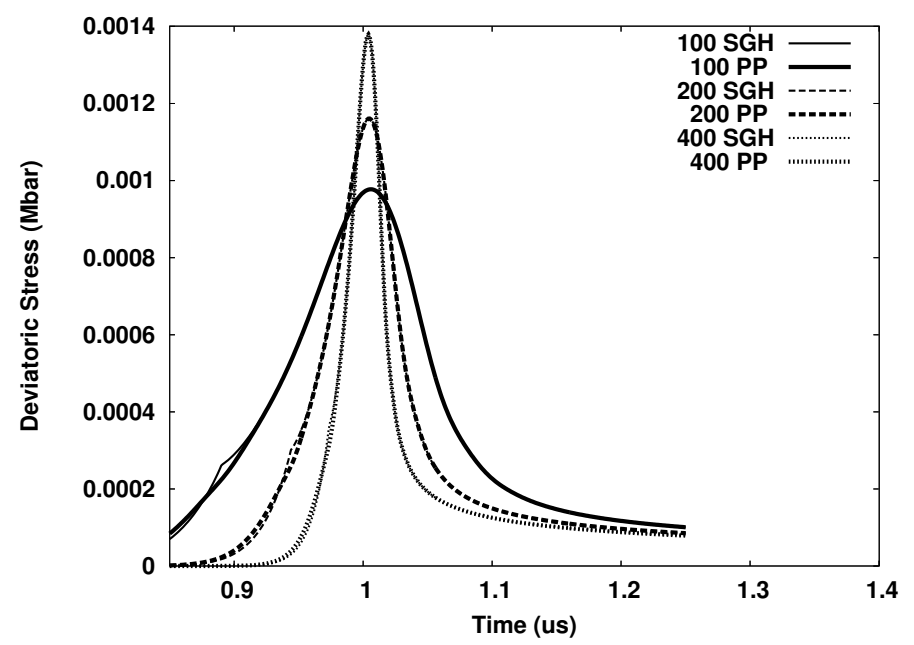

Figure 21: Deviatoric stress produced by the radial return algorithm and the radial return ODE overlaid. "SGH" is the SGH result while "PP" is the post-processed result, obtained by feeding the SGH results into the radial return ODE and PTW model. 
model still expects a finite rise time and the radial return method will use the strain rate, which will tend toward a Dirac delta, for its elastic projection step. Here, it has been shown that these singularities are integrable in this context. The radial return algorithm provides regularization, producing finite values for deviatoric stress. While the mathematical proof was for one very specific strain-rate hardening model, other evidence also provided in this article suggests that the result extends to more general cases, indicating that perhaps even for a multitude of strain-rate hardening models the regularization provided by radial return will be effective.

That is reassuring from a theoretical standpoint, however it was also shown here that the rise time, or numerical shock widths, required to capture a convergent deviatoric stress is exceedingly small compared to what contemporary hydrocodes could ever be expected to provide in application, and are also so small that they are not actually associated with a known physical process. In an effort toward remedying the non-convergence situation, it was demonstrated here that it may be possible to use the radial return ODE with a surrogate shock shape to augment calculations in the shock. Doing so could possibly circumvent the need for a highly refined mesh by replacing the SGH density rise with an extremely narrow surrogate, of converged width, in the radial return ODE. Put another way, instead of having the radial return method and yield stress model rely solely on the supervisory hydrocode's assessment of strain and strain rate for each cell, a shock detector could identify the cells containing the shock and, for those cells, appeal to a separate algorithm that relies on the ideas presented in this article to compute the deviatoric stress for the shocked cells.

That separate algorithm would assume a surrogate density profile as was done here, and use that as input into the radial return ODE, which would then be integrated numerically to obtain the deviatoric stress. The width of the surrogate could be adjusted to ensure that a value for the deviatoric stress is obtained that is within tolerance of a fully converged value, i.e., one which approximates the fully limited case of $w / D \rightarrow 0$. Since the numerical limiting procedure would only occur in the cells containing the shock, the additional computing time would be relatively minor.

For such an approach to work, an accurate and reliable shock locator would be required. Moreover, since shocks in numerical hydrocodes are spread out over multiple cells it would also be necessary to identify the cells surrounding but not containing the physical shock location so that the relatively high strain rates they experience due to numerical dissipation are filtered from the plasticity model. Similarly, it would become important for the shock locator to differentiate be- 
tween high strain rates that are part of a continuous phenomenon versus those that represent a shock.

The body of research dedicted to artificial viscosity activation and limiting could provide assistance with the latter while more recent work on highly accurate shock locaters [10] could provide assistance with the former. Developing that approach more fully is the subject of future research. This article provides motivation for why the pursuit of such a combination of technology is worthwhile and, at the same time, provides promise of a more accurate solution if the technology were implemented in a robust way.

\section{Acknowledgments}

The author thanks Ted Carney for his software in which the Gruneisen equation of state was implemented and verified, along with his implementation of the elastic-perfectly-plastic solution of [18]. The author also thanks the Materials Working Group at Los Alamos National Laboratory, in particular, Tom Canfield, and especially, Len Margolin for their insights. This work was performed under the auspices of the U.S. Department of Energy's NNSA by the Los Alamos National Laboratory, operated by Los Alamos National Security, LLC, under contract number DE-AC52-06NA25396. This article was released as LA-UR-13-28557.

\section{References}

[1] A.J. Barlow, P.L. Roe, A cell centred lagrangian godunov scheme for shock hydrodynamics, Computers and Fluids 46 (2011) 133-136.

[2] E. Caramana, D. Burton, M. Shashkov, P. Whalen, The construction of compatible hydrodynamics algorithms utilizing conservation of total energy, J. Comput. Phys. 146 (1998) 227-262.

[3] D. Grady, The shock wave profile, Shock Compression of Condens. Matter 955 (2007) 3-11.

[4] S. Hiermaier, Structures Under Crash and Impact: Continuum Mechanics, Discretization and Experimental Characterization, Springer Science+Business Media, LLC, 233 Spring Street, New York, NY, USA, 2008.

[5] P.H. Maire, A high-order cell-centered lagrangian scheme for twodimensional compressible fluid flows on unstructured meshes, J. Comput. Phys. 228 (2009) 2391-2425. 
[6] L.G. Margolin, E.C. Flower, Numerical simulation of plasticity at high strain rate, ASME Constitutive Modeling - Theory and Application (1991) 323334.

[7] L.G. Margolin, D.E. Vaughan, Traveling wave solutions for finite scale equations, Mech. Res. Commun. 45 (2012) 64-69.

[8] A. Molinari, G. Ravichandran, Fundamental structure of steady plastic shock waves in metals, J. Appl. Phys. 95 (2004) 1718-1732.

[9] N.R. Morgan, A dissipation model for staggered grid lagrangian hydrodynamics, Computers and Fluids 83 (2013) 48-57.

[10] E.M. Nelson, Reliable Estimation of Shock Position in Shock-Capturing Compressible Hydrodynamics Codes, Technical Report, 47th AIAA Aerospace Sciences Meeting, Orlando FL, 2009.

[11] W. Noh, Errors for calculations of strong shocks using an artificial viscosity and an artificial heat flux, J. Comput. Phys. 72 (1986) 78-120.

[12] A.D. Polyanin, V.F. Zaitsev, Handbook of Nonlinear Partial Differential Equations, Chapman and Hall/CRC Press, 2000 N.W. Corporate Blvd., Boca Raton, Florida 33431, 2004.

[13] D.L. Preston, D.L. Tonks, D.C. Wallace, Model of plastic deformation for extreme loading conditions, J. Appl. Physics 93 (2003) 211-220.

[14] W.J.M. Rankine, On the thermodynamic theory of waves of finite lognituinal disturbance, Philos. trans. Royal Society of London 146 (1869) 277-286.

[15] L. Sedov, Similarity and Dimensional Methods in Mechanics, CRC Press, Inc., 2000 N.W. Corporate Blvd., Boca Raton, Florida 33431, 2008.

[16] J.W. Swegle, D.E. Grady, Shock viscosity and the prediction of shock wave rise times, J. Appl. Phys. 58 (1985) 692-701.

[17] E.G. Tabak, A second-order godunov method on arbitrary grids, J. Comput. Phys. 124 (1996).

[18] H.S. Udaykumar, L. Tran, D.M. Belk, K.J. Vanden, An eulerian method for computation of multimaterial impact with eno shock-capturing and sharp interfaces, J. Comput. Phys. 186 (2003) 136-177. 
[19] J. VonNeumann, R. Richtmyer, A method for the numerical calculation of hydrodynamic shocks, J. of Appl. Phys. 21 (1950) 232-237.

[20] D.C. Wallace, Irreversible thermodynamics of overdriven shocks in solids, Phys. Rev. B 24 (1981) 5597-5605.

[21] D.C. Wallace, Nature of the process of overdriven shocks in metals, Phys. Rev. B 24 (1981) 5607-5615.

[22] W.N. Weseloh, S.P. Clancy, J.W. Painter, PAGOSA Phys. Manual, Technical Report, Los Alamos National Laboratory report, LA-14425-M, 2010.

[23] M.B. Zellner, G. Dimonte, T.C. Germann, J.E. Hammerberg, P.A. Rigg, G.D. Stevens, W.D. Turley, W.T. Buttler, Influence of shockwave profile on ejecta, 2009. 